\title{
Monitoring actuator failures for a large transport aircraft - the nominal case
}

\author{
Andras Varga \\ German Aerospace Center, DLR-Oberpfaffenhofen \\ Institute of Robotics and Mechatronics \\ D-82234 Wessling, Germany \\ Email: andras.varga@dlr.de
}

\begin{abstract}
We consider the problem of designing residual generators with least dynamical orders to solve actuator fault detection and isolation problems for a generic large transport aircraft. The main result of our analysis is the proof of feasibility of the complete isolation of all primary actuator/surface faults in the nominal case by using a minimal number of additional surface angle sensors. The analysis of the nominal case provides residual filter specifications (reference models) to be employed for robust synthesis of residual generators.
\end{abstract}

Keywords: Flight control, fault detection, actuator faults, structured residuals.

\section{INTRODUCTION}

The monitoring of primary actuator failures is of paramount importance for the safe operation of an aircraft, for a continuous situation awareness of pilots, and for the applicability of fault tolerant control (FTC) techniques to accommodate with various failure modes. The fault detection and isolation (FDI) of primary actuator failures which are relevant for the application of FTC techniques is illustrated in this paper for a generic transport aircraft with a full set of control surfaces/actuators.

The main goals of our analysis are: 1) Proving the feasibility of the FDI of all primary actuator faults; 2) Illustrating the potential of different approaches; 3) Determining achievable specifications for robust design; 4) Demonstrating the capabilities of recently developed design tools to solve complex monitoring problems. In what follows we provide some details on these goals and their achievement.

1) Several fault scenarios are of interest for actuator failures. The ability to detect single actuator faults is of major importance, being part of the aircraft control system certification requirements. Accordingly, a minimum requirement for a modern aircraft control system is that no single failure must lead to a catastrophic consequence. Simultaneous faults can also occur, especially in conjunction with surface damages. Their detection and isolation requires a more involved residual generation system and also the availability of a sufficiently large number of measurements. One of the main results of our study was to demonstrate the feasibility of FDI for a complete set of faults in a nominal case corresponding to a normal cruise flight.

2) The monitoring and diagnosis of actuator faults can be done at both component as well as at system level. The component level monitoring is traditionally used on present day aircraft and relies on the availability of surface

\footnotetext{
^ This work was supported via the ONERA-DLR Project IMMUNE: Intelligent Monitoring and Management of Unexpected Events.
}

angle sensors. Its capabilities to detect and even identify various fault types (e.g., loss of effectiveness, stuck or runaway faults, floating surface faults) have been discussed in (Varga, 2007b). However, this scheme has some intrinsic limitations, as for example, its inability to detect surface failures involving the loss of effectiveness. Also it does not work properly in the case of surface sensor failures. Therefore, monitoring all types of faults requires addressing the FDI problem (at least partially) using a system level approach. However, the system level approach has its own limitations due to the restricted number of available measurements, and therefore a full FDI is not possible unless additional surface sensors are used. An important result of our analysis is to show that the best FDI performance in terms of isolation capabilities and on-line implementation efforts are obtained when combining component level and system level fault monitoring techniques.

3) The results obtained for the nominal case consist of several residual generators and the corresponding fault-toresidual dynamics. The latter represent meaningful specifications for a more realistic design where the robustness aspects against parametric and operational point uncertainties as well as with respect to disturbances (e.g., wind gusts) are addressed. For this purpose, both optimal structured residual synthesis techniques (Varga, 2009a) as well as optimal model-matching techniques (Varga, 2005) are envisaged to be employed in a future study.

4) The employed computational tools represent enhancements of tools available in the FAULT DeTECTION Toolbox for MATLAB developed by the author (Varga, 2006), while the underlying algorithms are refined synthesis methods of least order residual generators (Varga, 2007a). It is worth mentioning, that due to the relatively large order of the underlying system, the reliable synthesis of low order residual generators was only possible by employing highly sophisticated computational techniques, like rational nullspace computations based on Kronecker-like forms or minimum dynamic covers based order reduction. 


\section{FAULT DETECTION AND ISOLATION PROBLEM}

Consider additive fault models described by input-output representations of the form

$$
\mathbf{y}(s)=G_{u}(s) \mathbf{u}(s)+G_{d}(s) \mathbf{d}(s)+G_{f}(s) \mathbf{f}(s),
$$

where $\mathbf{y}(s), \mathbf{u}(s), \mathbf{d}(s)$, and $\mathbf{f}(s)$ are Laplace-transformed vectors of the the $p$-dimensional system output vector $y(t)$, $m_{u}$-dimensional control input vector $u(t), m_{d}$-dimensional disturbance vector $d(t)$, and $m_{f}$-dimensional fault vector $f(t)$, respectively, and where $G_{u}(s), G_{d}(s)$ and $G_{f}(s)$ are the transfer-function matrices (TFMs) from the control inputs to outputs, disturbance inputs to outputs, and fault inputs to outputs, respectively. In a deterministic setting the disturbances are considered as unknown signals, while in a stochastic setting the disturbances are considered to be stochastic signals (e.g., white noise).

A linear residual generator (or fault detection filter) processes the measurable system outputs $y(t)$ and control inputs $u(t)$ and generates the residual signals $r(t)$ which serve for decision making on the presence or absence of faults. The input-output form of this filter is

$$
\mathbf{r}(s)=R(s)\left[\begin{array}{l}
\mathbf{y}(s) \\
\mathbf{u}(s)
\end{array}\right]
$$

where $R(s)$ is the TFM of the filter. For a physically realizable filter, $R(s)$ must be proper (i.e., only with finite poles) and stable (i.e., only with poles having negative real parts). The McMillan degree (or dynamic order) of $R(s)$ is the dimension of the state vector of a minimal state-space realization of $R(s)$. The dimension $q$ of the residual vector $r(t)$ depends on the fault detection problem to be solved. For example, for the detection of faults a single residual could be sufficient, but for isolating a fault among several possible faults a set of residuals grouped into a vector is needed.

The residual signal $r(t)$ in (2) generally depends via the system outputs $y(t)$ of all system inputs $u(t), d(t)$ and $f(t)$. The residual generation system, obtained by replacing in (2) $\mathbf{y}(s)$ by its expression from (1), is given by

$$
r(s)=R_{f}(s) \mathbf{f}(s)+R_{d}(s) \mathbf{d}(s)+R_{u}(s) \mathbf{u}(s)
$$

where the involved TFMs are defined as

$$
\left[R_{f}(s)\left|R_{d}(s)\right| R_{u}(s)\right]:=R(s)\left[\begin{array}{c|c|c}
G_{f}(s) & G_{d}(s) & G_{u}(s) \\
0 & 0 & I_{m_{u}}
\end{array}\right]
$$

For a successfully designed filter $R(s)$, the corresponding residual generation system is proper and stable and achieves specific fault detection requirements.

For a given detector with a $q \times\left(p+m_{u}\right)$ TFM $R(s)$, denote by $R_{f_{j}}^{i}(s)$ the $(i, j)$-th entry of the corresponding $R_{f}(s)$. We can define a $q \times m_{f}$ structure matrix $S$ corresponding to a residual set as follows:

$$
\begin{array}{lll}
S_{i j}= & 1 & \text { if } R_{f_{j}}^{i}(0) \neq 0 \\
S_{i j}= & -1 & \text { if } R_{f_{j}}^{i}(0)=0 \text { and } R_{f_{j}}^{i}(s) \neq 0 \\
S_{i j}= & 0 & \text { if } R_{f_{j}}^{i}(s)=0
\end{array}
$$

If $S_{i j}=1$ then we say that the fault $j$ is strongly detected in residual $i$. If $S_{i j}=-1$ then the fault $j$ is only weekly detected in residual $i$. The fault $j$ is not detected in residual $i$ if $S_{i j}=0$. We refer to the $i$-th row of $S$ as the $i$-th specification, while the $j$-th column of $S$ as the signature (or code) of fault $f_{j}$. This and related nomenclature used later is borrowed from (Gertler, 1998).
The following fault detection and isolation problem (FDIP) can be now formulated: Given a $q \times m_{f}$ structure matrix $S$ determine a bank of $q$ stable and proper scalar output residual generator filters

$$
\mathbf{r}_{i}(s)=R^{i}(s)\left[\begin{array}{l}
\mathbf{y}(s) \\
\mathbf{u}(s)
\end{array}\right], i=1, \ldots, q
$$

such that, for all $u(t)$ and $d(t)$ we have:

(i) $r_{i}(t)=0$ when $f_{j}(t)=0, \forall j$ with $S_{i j} \neq 0$;

(ii) $r_{i}(t) \neq 0$ when $f_{j}(t) \neq 0, \forall j$ with $S_{i j} \neq 0$.

In this formulation of the FDIP, each scalar output detector $R^{i}(s)$ achieves the $i$-th specification of the structure matrix $S$. The simplest case is to solve the fault detection problem (FDP), for $S=\left[\begin{array}{llll}1 & 1 & \cdots & 1\end{array}\right]$, using a scalar output detector. On the opposite side, to achieve the complete isolation of maximum $k$ simultaneous faults the choice $S=I_{k}$ is necessary. In many practical applications this strong isolation requirement can not be achieved due to the lack of sufficient number of measurements. If we can enforce a structure matrix with distinct fault signatures, then a so-called week isolation of faults is possible. For example, if for 3 fault inputs the structure matrix

$$
S=\left[\begin{array}{lll}
0 & 1 & 1 \\
1 & 0 & 1 \\
1 & 1 & 0
\end{array}\right]
$$

can be achieved, then the occurrence of a single fault $f_{j}$ can be detected if all residuals (excepting the $j$-th residual) are non-zero. More insight on how to specify fault signatures can be found in (Gertler, 1998, 2000).

Let $G_{f_{j}}(s)$ denote the $j$-th column of $G_{f}(s)$ and let $S$ be a given $q \times m_{f}$ structure matrix. We denote by $\bar{G}_{f}^{i}(s)$ the matrix formed from the columns of $G_{f}(s)$ whose column indices $j$ correspond to zero elements in the $i$-th specification. The solvability conditions of the FDIP are given by the following theorem (Gertler, 1998, p. 318):

Theorem 1. For the system (1) the FDIP with the given fault influence matrix $S$ is solvable if and only if for each $i=1, \ldots, q$, we have

$$
\operatorname{rank}\left[G_{d}(s) \bar{G}_{f}^{i}(s) G_{f_{j}}(s)\right]>\operatorname{rank}\left[G_{d}(s) \bar{G}_{f}^{i}(s)\right]
$$

for all $j$ such that $S_{i j} \neq 0$.

The standard approach to determine $R(s)$ is to design for each specification $i$, a detector $R^{i}(s)$ which generates the $i$-th residual signal $r_{i}(t)$, and thus represents the $i$-th row of $R(s)$. For this purpose, the nullspace method to design least order scalar output fault detection filters of (Varga, $2007 \mathrm{a}, \mathrm{b})$ can be applied. For each specification $i$, we redefine (temporarily) the fault components $f_{j}$ corresponding to $S_{i j}=0$ as disturbances and solve the FDP for the rest of faults whose indices $j$ correspond to $S_{i j} \neq 0$. In this way, we obtain a scalar output detector $R^{i}(s)$ which represents the $i$-th row of $R(s)$. The resulting global detector can be assembled as

$$
R(s)=\left[\begin{array}{c}
R^{1}(s) \\
\vdots \\
R^{q}(s)
\end{array}\right]
$$

We can also solve the FDIP in a stochastic setting, by considering the disturbances as white noise, with unit covariance. In this case, for each row $R^{j}(s)$ of the detector 
$R(s)$, we impose additionally the condition that in the absence of faults, the corresponding residual signal $r_{j}(t)$ is a white noise with unit covariance. If we denote $R_{d}^{j}(s)$ the $j$-th row of $R_{d}(s)$, then this condition amounts to ask that $R_{d}^{j}(s)$ is a co-inner function (i.e., $R_{d}^{j}(s)\left(R_{d}^{j}(-s)\right)^{T}=$ $1)$. Using the approach proposed by Nikoukhah (1994), we can update each row of $R(s)$, by replacing $R^{j}(s)$ by $\left(G_{o}^{j}(s)\right)^{-1} R^{j}(s)$, where $G_{o}^{j}(s) G_{i}^{j}(s)=R_{d}^{j}(s)$ is an outercoinner factorization of $R_{d}^{j}(s)$. The inverse $\left(G_{o}^{j}(s)\right)^{-1}$ of the stable and minimum-phase outer factor $G_{o}^{j}(s)$ is called a whitening filter.

The computational methods for the synthesis of residual generators rely on state space algorithms proposed in (Varga, 2007a), where the main computational ingredients are the computation of proper rational nullspace bases (Varga, 2003a, 2008a), order reduction by employing minimal dynamic covers based techniques (Varga, 2003b), and stable rational factorizations (Varga, 1998). For all these computations robust numerical software is available in the Descriptor Systems Toolbox Varga (2000). This software served as basis to implement a first version of a FAULT Detection Toolbox Varga (2006), where several tools are available to solve the main classes of fault detection problems. The most recent version of this toolbox is fully documented in Varga (2008b). A recent addition is a new function to compute the achievable structure matrix $S$ for a given system (1) based on a recently developed efficient and reliable numerical algorithm (Varga, 2009b).

\section{AIRCRAFT STATE SPACE MODEL WITH ADDITIVE FAULTS}

We consider a nominal linearized aircraft model with additive faults of the form

$$
\begin{aligned}
& \dot{x}(t)=A x(t)+B_{u} u(t)+B_{d} d(t)+B_{f} f(t) \\
& y(t)=C x(t)+D_{u} u(t)+D_{d} d(t)+D_{f} f(t)
\end{aligned}
$$

where $x(t)$ is the $n$-dimensional system state vector. The significance of independent variables is described in Appendix $\mathrm{A}$ and the numerical values of system matrices are given in (Varga, 2008c). The dimensions of vectors $x(t)$, $y(t), u(t), d(t)$ and $f(t)$, are respectively, $n=10, p=10$, $m_{u}=22, m_{d}=3$, and $m_{f}=8$. The corresponding TFMs of the model in (1) are

$$
\begin{aligned}
G_{u}(s) & =C(s I-A)^{-1} B_{u}+D_{u} \\
G_{d}(s) & =C(s I-A)^{-1} B_{d}+D_{d} \\
G_{f}(s) & =C(s I-A)^{-1} B_{f}+D_{f}
\end{aligned}
$$

The actuator and engine models are first order systems with the following transfer functions: $10 /(s+10)$ for each of two elevators, $0.5 /(s+0.5)$ for the stabilizer, $6.6 /(s+6.6)$ for each of four ailerons and ruder, $5 /(s+5)$ for each of 12 spoilers and $0.66 /(s+0.66)$ for each of two engines. The actuators system corresponds to a $22 \times 22$ block-diagonal TFM which has a state space realization of the form

$$
\begin{aligned}
\dot{x}_{a}(t) & =A_{a} x_{a}(t)+B_{a} u_{c}(t) \\
u(t) & =C_{a} x_{a}(t)+D_{a} u_{c}(t)
\end{aligned}
$$

where $x_{a}(t)$ is the state vector of dimension 22 and $u_{c}(t)$ contains the 20 deflection demands and the 2 thrust de- mands. The complete aircraft model resulted by coupling the actuators model at the system input is

$$
\begin{array}{r}
{\left[\begin{array}{c}
\dot{x}(t) \\
\dot{x}_{a}(t)
\end{array}\right]=\left[\begin{array}{cc}
A & B_{u} C_{a} \\
0 & A_{a}
\end{array}\right]} \\
\\
+\left[\begin{array}{c}
x(t) \\
x_{a}(t)
\end{array}\right]+\left[\begin{array}{c}
B_{u} D_{a} \\
B_{a} \\
0
\end{array}\right] d(t)+\left[\begin{array}{c}
B_{f} \\
0
\end{array}\right] f(t) \\
{\left[\begin{array}{c}
y(t) \\
\Pi u(t)
\end{array}\right]=\left[\begin{array}{cc}
C & D_{u} C_{a} \\
0 & \Pi C_{a}
\end{array}\right]} \\
{\left[\begin{array}{c}
x(t) \\
x_{a}(t)
\end{array}\right]+\left[\begin{array}{c}
D_{u} D_{a} \\
\Pi D_{a}
\end{array}\right] u_{c}(t)} \\
+\left[\begin{array}{c}
D_{d} \\
0
\end{array}\right] d(t)+\left[\begin{array}{c}
D_{f} \\
0
\end{array}\right] f(t)
\end{array}
$$

where $\Pi$ is an input selection matrix. This model has a state vector of dimension 32, 22 control inputs, and the number of measured variables can range between 10 and 18 (when all control surfaces corresponding to monitored actuators are provided with angle sensors). By suitably choosing $\Pi$, this model allows to study the case of an aircraft without angle sensors $(\Pi$ is an $0 \times 22$ empty matrix), or with an arbitrary set of angle sensors ( $\Pi$ is formed from up to 8 rows of the identity matrix $I_{22}$ ).

\section{SOLUTION OF FDIP - DETERMINISTIC CASE}

For the design of a fault monitoring system, we considered two cases. In the first case we assumed that no surface angle sensors are employed and we determined the best achievable signature structure which ensures a week isolation of single faults. In the second case, we add a minimal number of sensors which allows a better isolation of simultaneous faults.

To compute the achievable structure matrix $S$ for the aircraft model, we need to assess the weak/strong detectability of combinations of faults. For this purpose, for suitably chosen detectors $R^{i}(s)$, we set $S_{i j}=-1$ if $\left|R_{f_{j}}^{i}(0)\right| \leq 0.01$ and $R_{f_{j}}^{i}(s) \neq 0$. In the case, when no surface angle sensors are used, the achievable structure matrix computed with the algorithm of Varga $(2009 \mathrm{~b})$ is the $55 \times 8$ matrix

$$
S=\left[\begin{array}{rrrrrrrr}
1 & 1 & 1 & 1 & 1 & 1 & 1 & 1 \\
0 & 1 & 1 & 1 & 1 & 1 & 1 & 1 \\
0 & 0 & 1 & 1 & 1 & 1 & 1 & 1 \\
0 & 0 & 0 & 0 & 0 & 0 & 0 & 1 \\
0 & 0 & 1 & 1 & 1 & 1 & 1 & 0 \\
0 & -1 & 0 & -1 & 1 & 1 & 1 & 1 \\
0 & -1 & 0 & -1 & 1 & 1 & 1 & 0 \\
0 & -1 & -1 & 0 & 1 & 1 & 1 & 1 \\
& & & \vdots & & & & \\
1 & 1 & 1 & 0 & 1 & 1 & 1 & 0 \\
1 & 1 & 1 & 1 & 0 & 1 & 1 & 1 \\
1 & 1 & 1 & 1 & 0 & 0 & 0 & 1 \\
1 & 1 & 1 & 1 & 0 & 0 & 0 & 0 \\
1 & 1 & 1 & 1 & 0 & 1 & 1 & 0 \\
1 & 1 & 1 & 1 & 1 & 0 & 1 & 1 \\
1 & 1 & 1 & 1 & 1 & 0 & 1 & 0 \\
1 & 1 & 1 & 1 & 1 & 1 & 0 & 1 \\
1 & 1 & 1 & 1 & 1 & 1 & 0 & 0 \\
1 & 1 & 1 & 1 & 1 & 1 & 1 & 0
\end{array}\right]
$$


where, as it can be observed, there are many lines contain ing negative entries corresponding to week detectability o the faults. There are 47 strongly detectable specification: which can be used as basis for selecting an optimal desirec set of specifications for the sensor free case.

For example, the signature structure

$$
S_{1}=\left[\begin{array}{llllllll}
1 & 1 & 1 & 1 & 1 & 1 & 1 & 1
\end{array}\right]
$$

can be used to perform fault detection at system leve (e.g., to complement an already existing component leve monitoring). The resulting detector has order 5 and the step responses from the faults can be seen in Fig. 1 Thus, strong fault detection can be achieved without an: additional surface angle sensor information.

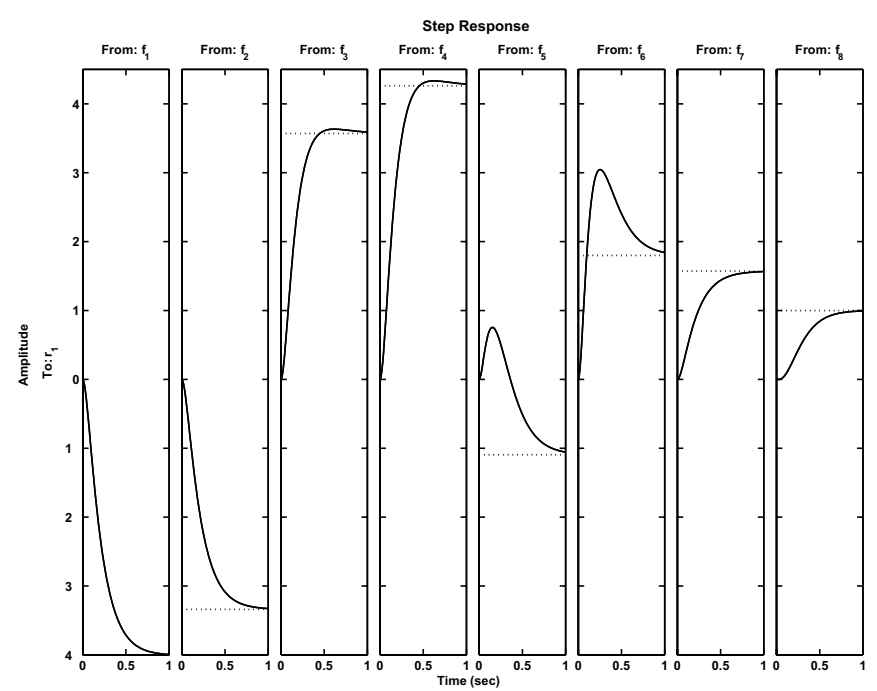

Fig. 1. Step responses from the faults

It is possible to achieve the isolation of all single faults using the following specification

$$
S_{2}=\left[\begin{array}{llllllll}
0 & 1 & 1 & 1 & 1 & 0 & 1 & 0 \\
1 & 0 & 1 & 1 & 0 & 1 & 1 & 0 \\
1 & 1 & 0 & 1 & 1 & 0 & 1 & 0 \\
1 & 1 & 1 & 0 & 0 & 1 & 1 & 0 \\
1 & 1 & 1 & 1 & 0 & 0 & 0 & 0 \\
0 & 0 & 0 & 0 & 0 & 0 & 0 & 1
\end{array}\right]
$$

which ensures the strong isolation of ruder faults (inde pendently of other faults) and the weak isolation of the rest of faults occurring one at a time. The resulting banl of 6 detectors has a global order 32, where the six scala: output detectors have the orders: $\{6,6,6,6,4,4\}$. In Fig. : we present the step response of the fault detection system from which the achieved fault signature can be easil! checked.

By employing angle sensors on the two outer ailerons anc on the stabilizer, a better isolation of simultaneous fault: can be achieved using the specification

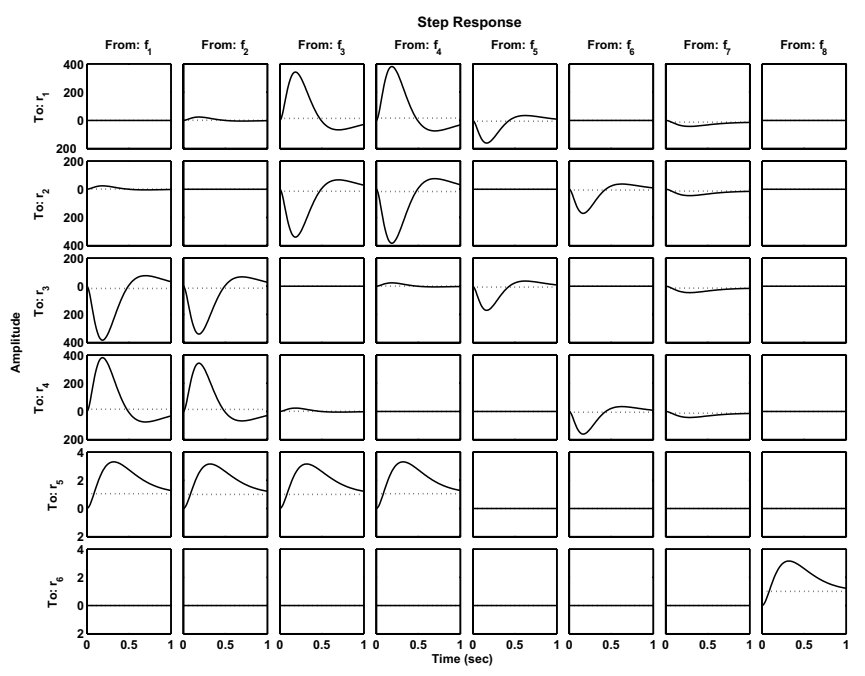

Fig. 2. Step responses from the faults

$$
S_{3}=\left[\begin{array}{llllllll}
1 & 0 & 0 & 0 & 0 & 0 & 0 & 0 \\
0 & 0 & 1 & 0 & 1 & 1 & 0 & 0 \\
0 & 1 & 0 & 0 & 1 & 1 & 0 & 0 \\
0 & 0 & 0 & 1 & 0 & 0 & 0 & 0 \\
0 & 1 & 1 & 0 & 0 & 1 & 0 & 0 \\
0 & 1 & 1 & 0 & 1 & 0 & 0 & 0 \\
0 & 0 & 0 & 0 & 0 & 0 & 1 & 0 \\
0 & 0 & 0 & 0 & 0 & 0 & 0 & 1
\end{array}\right]
$$

This provides strong fault isolation for the outer ailerons, stabilizer and ruder (the faults can be isolated if they occur simultaneously or not with other faults) and weak fault isolation for left/right inner ailerons and left/right elevators. The resulting bank of 8 detectors has a global order 27, where the six scalar output detectors have the orders: $\{1,5,5,1,5,5,1,4\}$. Note that the first order detectors correspond to a component level monitoring and the resulting detectors are the same as when considering actuator/surface systems alone with first order dynamics. In Fig. 3 we present the step response of the fault detection system, from which the achieved fault signature can be easily checked.

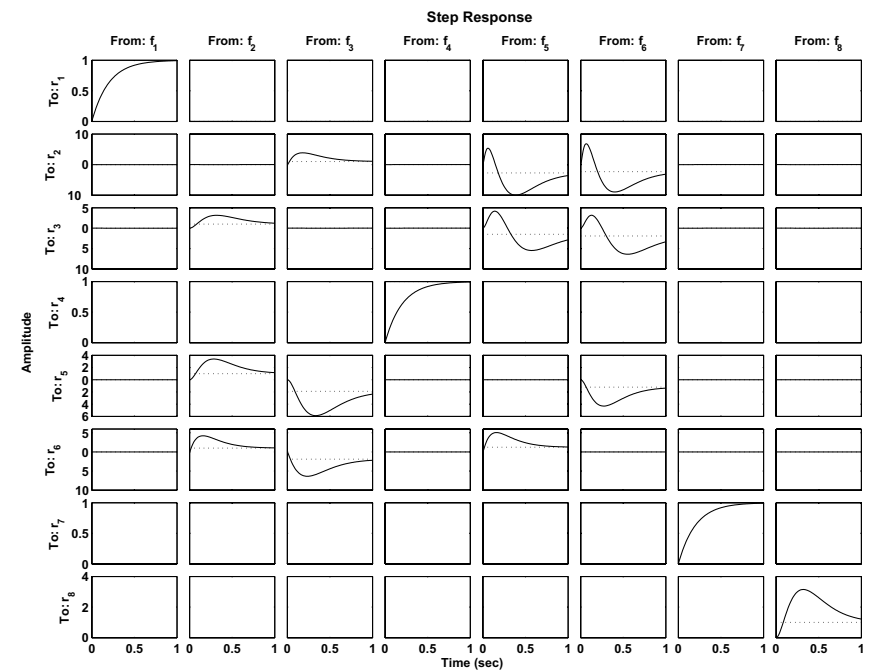

Fig. 3. Step responses from the faults 
Strong isolation of all faults, i.e. the specification $S_{4}=I_{8}$, can be achieved with 7 angle sensors and a detector of global order 9 , or with 8 sensors and a detector of global order 8. This last case corresponds to employing only local monitoring and due to the employed least order synthesis based approach (Varga, 2007a), can be completely recovered using an unique high order system model.

\section{SOLUTION OF FDIP - STOCHASTIC CASE}

It is interesting to compare two cases for the synthesis of residual generators for fault detection: first, when we completely ignore the noise inputs in the synthesis of the residual generator, and second, when we use additionally a whitening filter. In both cases, the resulting filter has order 4. For the first case, we show in Fig. 4 the time response of the residual signal to a right outer aileron fault represented by an unit step at $t=6.6 \mathrm{sec}$ and white noise disturbance inputs of covariance 0.1 .

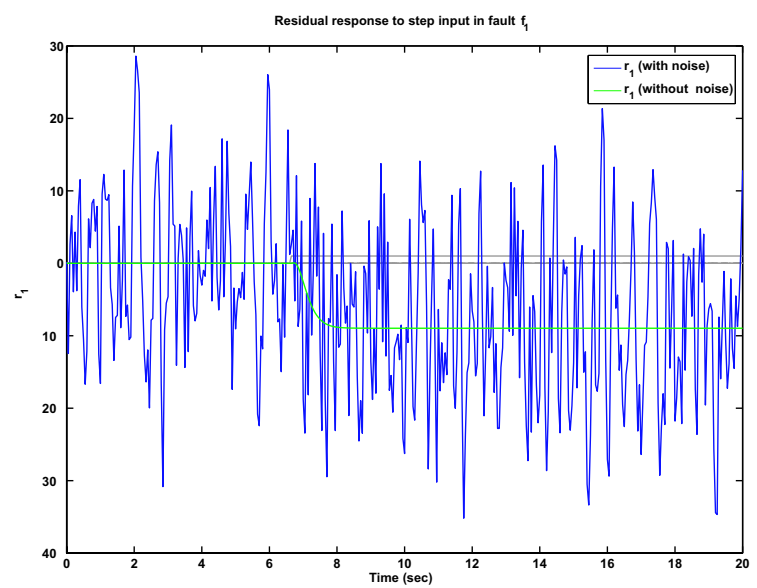

Fig. 4. Residual $r_{1}$ response to a unit step in $f_{1}$

Contrasting with this, in the second case a strong filtering effect can be observed in Fig. 5, where the same inputs are used. This solution is practically the same as that obtained by using the recently proposed $\mathcal{H}_{-} / \mathcal{H}_{2}$ and $\mathcal{H}_{-} / \mathcal{H}_{\infty}$ techniques (Varga, 2009a).

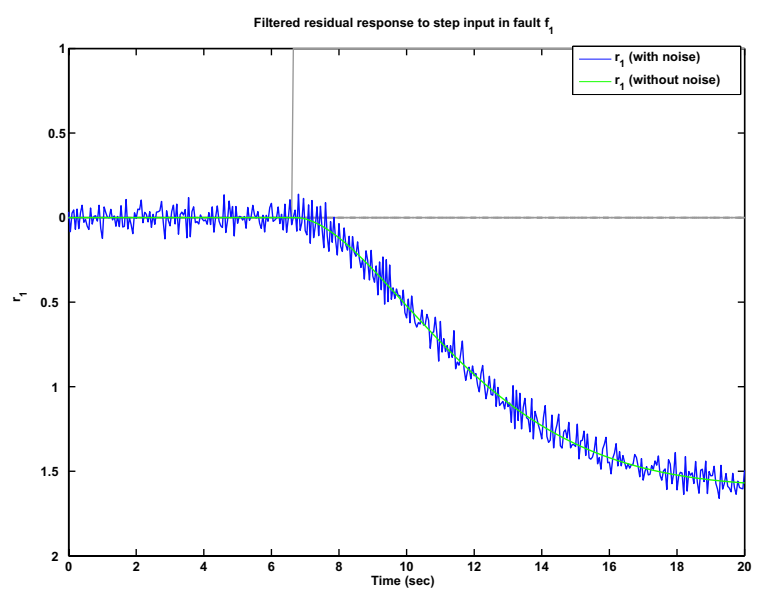

Fig. 5. Filtered residual $r_{1}$ response to a unit step in $f_{1}$

We can now apply the whitening filters to each residual generator output corresponding to the signature structure
$S_{2}$. The resulting total order of the detector is 25 and the orders of individual detectors are $\{5,5,5,5,3,2\}$. Note that this order is less than the global order, 32, of the corresponding residual generator obtained in the deterministic setting. The time responses for three single faults in $f_{1}$, $f_{3}$, and $f_{8}$ are shown in Fig. 6. Observe that the achieved fault signatures (columns 1,3 , and 8 of $S_{2}$ ) can be easily read out form the corresponding time responses.
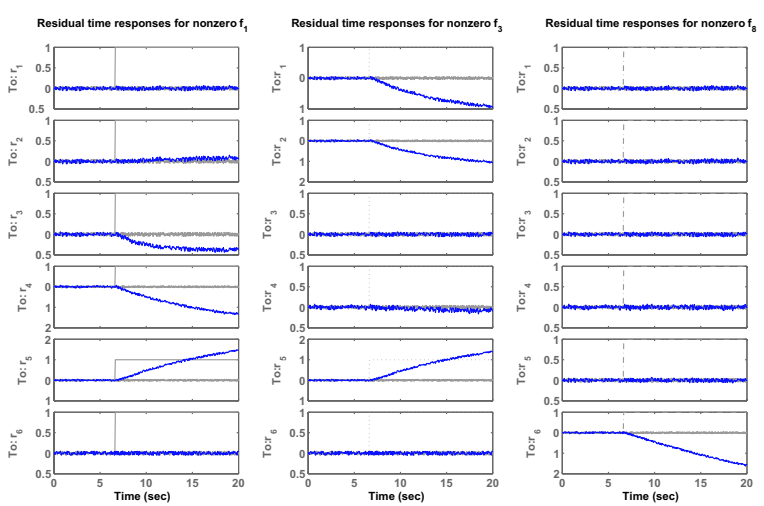

Fig. 6. Residual time responses to unit steps in single faults

The global detector corresponding to $S_{3}$ has order 17, with the individual detectors having orders: $\{1,3,3,1,3,3,1,2\}$. As before, the first order detectors correspond to a component level monitoring.

The main difficulty of using the stochastic setting is that the dynamics of the detector is determined by the zeros of the outer factors, and thus is fixed. This led to a poor dynamics of the fault detection system in always all cases. Thus, although the achieved orders are generally smaller than for the equivalent deterministic problems, still the detectors are more difficult to be used in safety critical applications like an aircraft.

\section{CONCLUSIONS}

The combination of component and system level fault monitoring allows the practical solution of the FDIP for 8 primary actuator faults in both deterministic and stochastic settings. We have shown that 3 surface angle sensors are sufficient for this purpose. All residual generators have least orders being obtained using recently developed algorithms based on minimal dynamic cover techniques (Varga, 2007a). All computations have been done using recently developed numerical software tools included in the current version (V0.8) of the FAULT Detection Toolbox of DLR. The computed detectors for the nominal case will serve as specifications for a more realistic design of robust residual generators.

Two aspects are worth of mentioning to illustrate the new features of the performed synthesis. The first aspect is the use of least order synthesis techniques, which allow to obtain detectors of acceptable complexity. Note that without this feature, the generic order of each individual detector is the system order (see (Hou and Müller, 1994) for examples) and thus not acceptable for larger order systems. For example, for the 6 detectors used to achieve the signature $S_{2}$ in both deterministic and stochastic 
settings, the expected order is $6 \times 32=192$, which is certainly not appropriate for on-line implementations.

The second aspect is determined by the high reliability of the underlying computational algorithms and of the corresponding software. This feature allows to manipulate a single, relatively large order system representation to achieve a seamless transition between component and system level monitoring. In the extreme case when all angle sensors are provided, the computed results are the same as individually designed detectors for each actuator.

\section{REFERENCES}

J. Gertler. Fault Detection and Diagnosis in Engineering Systems. Marcel Dekker, New York, 1998.

J. Gertler. Designing dynamic consistency relation for fault detection and isolation. Int. J. Control, 73:720732, 2000.

M. Hou and P. C. Müller. Fault detection and isolation observers. Int. J. Control, 60:827-846, 1994.

R. Nikoukhah. Innovations generation in the presence of unknown inputs: application to robust failure detection. Automatica, 30:1851-1867, 1994.

A. Varga. A Descriptor Systems toolbox for MAtlab. Proc. CACSD'2000 Symposium, Anchorage, Alaska, 2000.

A. Varga. On computing least order fault detectors using rational nullspace bases. Proc. of IFAC Symp. SAFEPROCESS'2003, Washington D.C., 2003a.

A. Varga. Reliable algorithms for computing minimal dynamic covers. Proc. of CDC'2003, Maui, Hawaii, $2003 \mathrm{~b}$

A. Varga. Numerically reliable methods for optimal design of fault detection filters. Proc. of CDC'05, Seville, Spain, 2005.

A. Varga. A fault detection toolbox for Matlab. Proc. of CACSD'06, Munich, Germany, 2006.

A. Varga. On designing least order residual generators for fault detection and isolation. Proc. 16th Internat. Conf. on Control Systems and Computer Science, Bucharest, Romania, pp. 323-330, 2007a.

A. Varga. Fault detection and isolation of actuator failures for a large transport aircraft. Proc. First CEAS European Air and Space Conference, Berlin, Germany, 2007b.

A. Varga. On computing nullspace bases - a fault detection perspective. Proc. IFAC 2008 World Congress, Seoul, Korea., pp. 6295-6300, 2008a.

A. Varga. Linear FDI-Techniques and Software Tools. FAult Detection Toolbox V0.8 - Technical Documentation IB 515-08-18, German Aerospace Center (DLR), Institute of Robotics and Mechatronics, 2008b.

A. Varga. Design of residual generator filters for monitoring actuator faults for the IMMUNE benchmark - the nominal case. Technical Report IB 515-08-49, German Aerospace Center (DLR), Institute of Robotics and Mechatronics, 2008c.
A. Varga. General computational approach for optimal fault detection. Proc. SAFEPROCESS'09, Barcelona, Spain, 2009a.

A. Varga. On computing achievable fault signatures. Proc. SAFEPROCESS'09, Barcelona, Spain, 2009b.

A. Varga. Computation of coprime factorizations of rational matrices. Lin. Alg. \& Appl., 271:83-115, 1998.

\section{Appendix A. SYSTEM VARIABLES}

$$
x=\left(\begin{array}{l}
\text { roll rate } \\
\text { pitch rate } \\
\text { yaw rate } \\
\text { first component of quaternion } \\
\text { second component of quaternion } \\
\text { third component of quaternion } \\
\text { fourth component of quaternion } \\
\text { ground speed } X \text { axis } \\
\text { ground speed } Y \text { axis } \\
\text { ground speed } Z \text { axis }
\end{array}\right)
$$$$
y=\left(\begin{array}{l}
\text { roll angle } \\
\text { pitch angle } \\
\text { yaw angle } \\
\text { angle of attack } \\
\text { angle of sideslip } \\
\text { flight path angle } \\
\text { roll rate } \\
\text { pitch rate } \\
\text { yaw rate } \\
\text { true airspeed }
\end{array}\right)
$$$$
u=\left(\begin{array}{l}
\text { right outer aileron deflection } \\
\text { right inner aileron deflection } \\
\text { spoiler }_{1} \text { deflection } \\
\quad \\
\text { spoiler } 12 \text { deflection } \\
\text { left inner aileron deflection } \\
\text { left outer aileron deflection } \\
\text { right elevator deflection } \\
\text { stabilizer trim angle } \\
\text { left elevator deflection } \\
\text { rudder deflection } \\
\text { left engine thrust } \\
\text { right engine thrust }
\end{array}\right)
$$$$
d=\left(\begin{array}{l}
\text { wind speed } X \text { axis } \\
\text { wind speed } Y \text { axis } \\
\text { wind speed } Z \text { axis }
\end{array}\right)
$$$$
f=\left(\begin{array}{l}
\text { right outer aileron fault } \\
\text { right inner aileron fault } \\
\text { left inner aileron fault } \\
\text { left outer aileron fault } \\
\text { right elevator fault } \\
\text { left elevator fault } \\
\text { stabilizer fault } \\
\text { ruder fault }
\end{array}\right)
$$ 